\title{
Gamificação Aplicada a Educação: Um Mapeamento Sistemático da Literatura
}

Júlia de Avila dos Santos - Universidade Federal do Rio Grande (FURG) juliaavila.santos@gmail.com

André Luis Castro de Freitas - Universidade Federal do Rio Grande (FURG) dmtalcf@furg.br

Resumo: Gamificação é um processo que utiliza elementos de jogos em aplicações nãojogo, tal que a área da educação torna-se uma área com elevado potencial de aproveitamento, uma vez que alcança promover a motivação e o engajamento dos alunos. Realizou-se um mapeamento sistemático da literatura, analisando artigos que abordaram a gamificação aplicada a educação. Grande parte dos trabalhos implementam a gamificação inseridos no contexto de softwares educacionais. A partir desses estudos, tornou-se possivel compreender que o problema de estimulo dos alunos em sala de aula é constante, com isso, diferentes pesquisas já estão analisando o beneficio da gamificação e incorporando-a no processo de ensino-aprendizagem.

Palavras-chave: gamificação; educação; mapeamento sistemático da literatura.

\section{Gamification Applied to Education: A Systematic Review of the Literature}

\begin{abstract}
Gamification is a process that uses game elements in non-game applications. This concept makes the area of education become an area with high potential for achievement as it promotes the motivation and engagement of students. In the article a systematic review of the literature was done, analyzing articles that approached gamification applied to education. It was noticed that a great part of the works implements gamification inserted in the context of educational software. From these studies, it became possible to understand that the problem of student stimulation in the classroom is constant, with this, different researches are already analyzing the benefit of gamification and incorporating it into the teaching-learning process.
\end{abstract}

Keywords: gamification; education; systematic review of the literature.

\section{Considerações Iniciais}

A gamificação surgiu com a influência, popularidade e popularização dos jogos e também da cultura digital, mas não necessita obrigatoriamente das tecnologias digitais para ser aplicada. A gamificação, em conceito geral, consiste na utilização de elementos e características dos jogos fora do contexto do mesmo, com a finalidade de contribuir para a resolução de algum problema. Essa técnica já vem sendo empregada em áreas como o marketing, para auxiliar, por exemplo, no engajamento e fidelização de clientes (Zichermann et. al., 2011). Nos últimos anos, observou-se um crescente interesse pelo aproveitamento no uso desse processo na área da educação com o objetivo de motivar e engajar os alunos nas realizações de suas atividades (Kapp, 2012; Domínguez et. al., 2013). No entanto, para entender a motivação originada pelos elementos de jogos é necessário identificar quais desses elementos são mais aceitos e benéficos na sala de aula. Por meio desse mapeamento sistemático da literatura, espera-se reunir informações que auxiliem na implementação da gamificação, melhorando os resultados obtidos e 
consequentemente aumentando a disseminação desse conceito, expondo, ainda, aplicações computacionais, com a finalidade de promover a discussão e apresentação sobre esse fenômeno.

Um mapeamento sistemático da literatura, assim como outros tipos de estudos, é uma forma de pesquisa que utiliza como fonte de dados à literatura sobre determinado tema. Esse tipo de investigação disponibiliza resultados específicos e detalhados por meio da análise de conteúdo e qualidade do material pesquisado (Kitchenham et. al., 2004) e, ainda, resume as evidências relacionadas a uma determinada questão mediante a aplicação de métodos explícitos e sistematizados de busca (Mancini et. al., 2007). O objetivo geral desse mapeamento sistemático é construir uma visão dos estudos que estão sendo conduzidos sobre gamificação aplicada à área de educação. Além dessa finalidade, o mapeamento tem dois objetivos específicos: 1) Localizar trabalhos relacionados ao assunto gamificação na área da educação; e 2) Desenvolver conhecimento sobre a fundamentação teórica da gamificação.

Dessa forma, um mapeamento sistemático da literatura proporciona uma visão geral do que tem sido investigado em determinado contexto. Por outro lado, se reconhece que qualquer estudo pode sofrer ameaças a sua qualidade, tal que se faz necessário que os pesquisadores garantam um processo claro, definindo os objetivos, as questões de pesquisa, os critérios de exclusão e inclusão e as bases de dados antes de todo o processo de busca.

$\mathrm{O}$ artigo está dividido conforme segue: na seção 2. O Processo de Revisão da Literatura define-se as etapas da revisão e seu desenvolvimento; na seção 3. Análise e discussão apresenta-se a construção dos resultados das análises dos artigos. Após seguem as considerações finais.

\section{O Processo de Revisão da Literatura}

De acordo com Petersen et. al. (2008) em uma revisão da literatura para as análises e resultados serem sucintos e oferecer credibilidade devem apresentar cinco passos fundamentais a serem seguidos: 1) Definição de questões de pesquisa; 2) Realização da busca; 3) Triagem dos documentos; 4) Determinação de classes; e 5) Extração de dados e mapeamento.

Analisando que as questões de pesquisa devem identificar o objetivo da revisão e, ainda, delimitar o alvo da busca, é importante mencionar que este mapeamento é parte de um projeto em andamento onde os autores investigam o uso de gamificação na educação, analisando técnicas de gamificação (elementos de jogos usados com o intuito de engajar os alunos ao aplicativo não-jogo) em Ambientes Virtuais de Aprendizagem (AVAs). Com isso, as seguintes questões foram elaboradas:

Questão 1: As técnicas de gamificação auxiliam no processo de ensino e aprendizagem?

Questão 2: Quais são as técnicas de gamificação utilizáveis no processo de ensino e aprendizagem?

Primeiramente, foram selecionadas as bases de dados eletrônicas que são relevantes para a área de pesquisa da revisão, seguido a escolha de termos ou palavras chaves para a busca. Nessa revisão, optou-se que a palavra-chave seria somente gamificação, no idioma português e gamification, no idioma inglês. Porém, os critérios de inclusão e exclusão (próximo passo), avaliaram se o artigo está relacionado com as questões de pesquisa e inserido na área de educação. Foram selecionados seis repositórios, conforme apresentados na Tabela 1, cuja escolha aconteceu pelo 
reconhecimento e acesso gratuito dos mesmos. É importante em uma revisão apresentar a data de busca realizada, tal que, para esse trabalho, as buscas ocorreram durante os meses de outubro de 2016 e fevereiro de 2017.

Tabela 1. Repositórios utilizados na revisão da literatura

\begin{tabular}{|l|l|}
\hline \multicolumn{1}{|c|}{ Repositórios } & \multicolumn{1}{|c|}{ Endereço } \\
\hline $\begin{array}{l}\text { Congresso Brasileiro de Informática na Educação } \\
\text { (CBIE) }\end{array}$ & http://www.br-ie.org/pub/index.php/wcbie/index \\
\hline $\begin{array}{l}\text { Revista Novas Tecnologias na Educação } \\
\text { (RENOTE) }\end{array}$ & http://seer.ufrgs.br/renote/ \\
\hline Revista Brasileira de Informática na Escola (RBIE) & http://www.br-ie.org/pub/index.php/rbie \\
\hline $\begin{array}{l}\text { Simpósio Brasileiro de Informática na Educação } \\
\text { (SBIE) }\end{array}$ & http://www.br-ie.org/pub/index.php/sbie \\
\hline Workshop de Informática na Escola (WIE) & http://www.br-ie.org/pub/index.php/wie \\
\hline Scientific Electronic Library Online (Scielo) & http://www.scielo.org/php/index.php \\
\hline
\end{tabular}

$\mathrm{Na}$ primeira etapa, buscando pesquisas com as palavras-chave definidas, foi obtido um resultado de 71 trabalhos. No próximo passo, as seleções dos trabalhos foram baseadas nos critérios de inclusão e exclusão conforme o objetivo do estudo.

Tabela 2. Critérios de inclusão e exclusão de artigos

\begin{tabular}{|l|l|}
\hline \multicolumn{1}{|c|}{ Critérios de Inclusão } & \multicolumn{1}{c|}{ Critérios de Exclusão } \\
\hline $\begin{array}{l}\text { 1. Apresentam relação com a educação. } \\
\text { 2.Apresentam relação com abordagens práticas na } \\
\text { gamificação. }\end{array}$ & $\begin{array}{l}\text { 1. Aqueles que não apresentam relação com as } \\
\text { questões de pesquisa e objetivos do projeto. } \\
\text { 2. Revisões sistemáticas da literatura e } \\
\text { mapeamento de estudos. } \\
\text { 3. Trabalhos que apresentam propostas de } \\
\text { gamificação sem resultados e validações. } \\
\text { 4. Versões anteriores de trabalhos selecionados. }\end{array}$ \\
\hline
\end{tabular}

Durante a primeira seleção dos trabalhos, que ocorreu por meio da avaliação dos títulos, dos resumos e palavras-chaves, aplicando os critérios, foram selecionadas 42 pesquisas. Logo após, para a segunda seleção, uma leitura da introdução e conclusão, e novamente aplicando os critérios de inclusão e os de exclusão, 22 trabalhos foram selecionados. Ressaltando que em alguns artigos se fez necessário a leitura de outras partes do texto para obter resultados melhores. Logo a seguir a tabela com as relações dos repositórios, e resultados gerais, da primeira e segunda etapa de seleção.

Tabela 3. Estudos encontrados no passo de triagem de documentos

\begin{tabular}{|l|c|c|c|}
\hline \multicolumn{1}{|c|}{ Repositório } & Resultado & $1^{\text {o }}$ seleção & $2^{\text {o }}$ seleção \\
\hline CBIE & 19 & 10 & 5 \\
\hline RENOTE & 9 & 5 & 3 \\
\hline RBIE & 2 & 2 & 2 \\
\hline SBIE & 29 & 18 & 8 \\
\hline Scielo & 7 & 3 & 0 \\
\hline WIE & 5 & 4 & 4 \\
\hline Total & $\mathbf{7 1}$ & $\mathbf{4 2}$ & $\mathbf{2 2}$ \\
\hline
\end{tabular}


Seguindo os passos, logo após a segunda seleção foram identificadas categorias. Essas categorias foram elaboradas avaliando as questões de pesquisa do mapeamento sistemático da literatura e seus objetivos, relembrando que esse estudo faz parte de um projeto em andamento dos autores. Cada artigo foi classificado de acordo com o objetivo principal para a pesquisa em relação à gamificação. Mas é importante ressaltar, que esse argumento não justifica que alguns artigos não sejam de uma ou mais categorias.

1. Estudo Empírico: estudos que os autores afirmaram que as técnicas de gamificação podem contribuir para o processo de aprendizagem, com a apresentação de evidências empíricas.

2. Referencial Teórico: estudos com o objetivo de apresentar a gamificação aplicada educação.

3. Métodos: estudos que apresentaram recursos, técnicas, abordagens e estratégias de gamificação para o processo de aprendizagem.

$\mathrm{Na}$ próxima seção serão analisados e discutidos os artigos selecionados na revisão, que por meio desses foi possível um entendimento dos assuntos relacionados e o que estava sendo trabalhado nas pesquisas. Assim, foram selecionados no repositório CBIE os artigos: Maekawa et. al. (2015), Pedro et. al. (2016), Faria et. al. (2016), Nagai et. al. (2016a) e Silva et. al. (2016). No repositório RENOTE: Fardo (2013), Klock et. al. (2014) e Klock et. al. (2015). No RBIE os artigos: Lopes et. al. (2015) e Colpani et. al. (2016). Já no repositório SBIE: Andrade et. al. (2013), Falcão et. al. (2014), Seixas et. al. (2014), Neto et. al. (2015), Ramos et. al. (2015), da Silva et. al. (2016), de Freitas et. al. (2016) e Raposo et. al. (2016). E no repositório WIE os artigos: Ferreira et. al. (2016), Toda et. al. (2016), Almeida et. al. (2016) e Nagai et. al. (2016b).

\section{Análise e Discussão}

Ainda segundo Petersen et. al. (2008), o mapeamento apresenta os resultados da revisão expondo na forma de mapas, gráficos e tabelas, junto com as discussões e conclusões. Após as análises dos estudos foi possível construir a classificação de acordo com as categorias definidas. Percebe-se que a maioria dos artigos selecionados foram de estudo empírico: um estudo de caso em que os autores aplicaram uma metodologia das técnicas de gamificação para validar a contribuição no processo de aprendizagem, no engajamento, motivação e melhoria de habilidades do aluno. Diferente dos outros, o trabalho de Fardo (2013) não foi classificado na primeira categoria, pois o autor não realiza um estudo de caso próprio, ele apenas descreve um relato sobre a utilização da gamificação em um ambiente de aprendizagem. Registra-se que todo o artigo que tratava de um estudo empírico sempre aborda referências sobre a gamificação, aplicada a educação, não como foco, mas como introdução, por esse motivo não foram classificados na segunda categoria: Referencial Teórico. Cabe ressaltar que nem todo artigo com o estudo empírico apresentou uma referência de abordagem de método, por isso a separação das categorias.

Tabela 4. Quantidade de estudos em cada categoria

\begin{tabular}{|l|c|}
\hline \multicolumn{1}{|c|}{ Categorias } & Quantidade de estudos \\
\hline 1. Estudo Empírico & 21 \\
\hline 2. Referencial Teórico & 5 \\
\hline 3. Métodos & 8 \\
\hline
\end{tabular}


De acordo com os dados encontrados, é possível demonstrar que as pesquisas de gamificação são recentes, observando, ainda, que os artigos analisados possuem o foco na educação, com as questões de pesquisa e objetivos da revisão. Dos 22 estudos selecionados, 12 são do ano de 2016, 5 são de 2015, 3 de 2014, e os 2 mais antigos do ano de 2013.

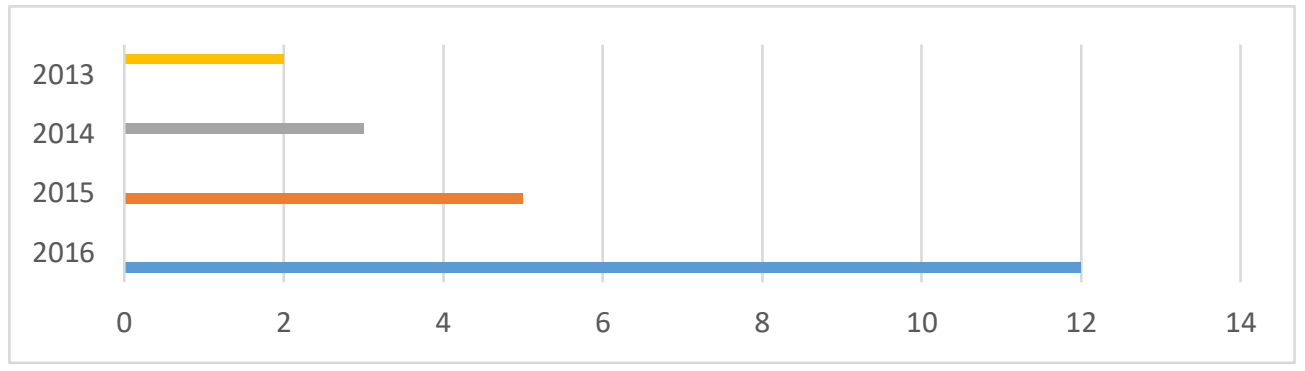

Figura 1. Estudos classificados pelo ano de publicação

Observando a Figura 2 é possível perceber como foi construída a base empírica dos artigos, nota-se que na maioria das pesquisas foram implementadas ferramentas com elementos de jogos para o estudo de caso. O artigo de Nagai et. al. (2016a) foi classificado como ferramenta desenvolvida, mas é importante mencionar que o sistema só foi utilizado para acompanhar as pontuações obtidas, medalhas e níveis alcançados em cada atividade na sala de aula. Seguindo, cinco artigos utilizaram softwares educacionais online que utilizam as técnicas de gamificação. E ainda, quatro trabalhos colocaram a prática na gamificação na sala de aula sem o uso da tecnologia, modificando a didática. Analisou-se que, apenas, um artigo realizou um estudo de caso com o foco nos elementos de jogos mais consagrados e aceitos pelos jogadores. Nesse artigo, Lopes et. al. (2015) no processo de validação dos dados aplicaram formulários compartilhados em redes sociais e comunidades de jogo.

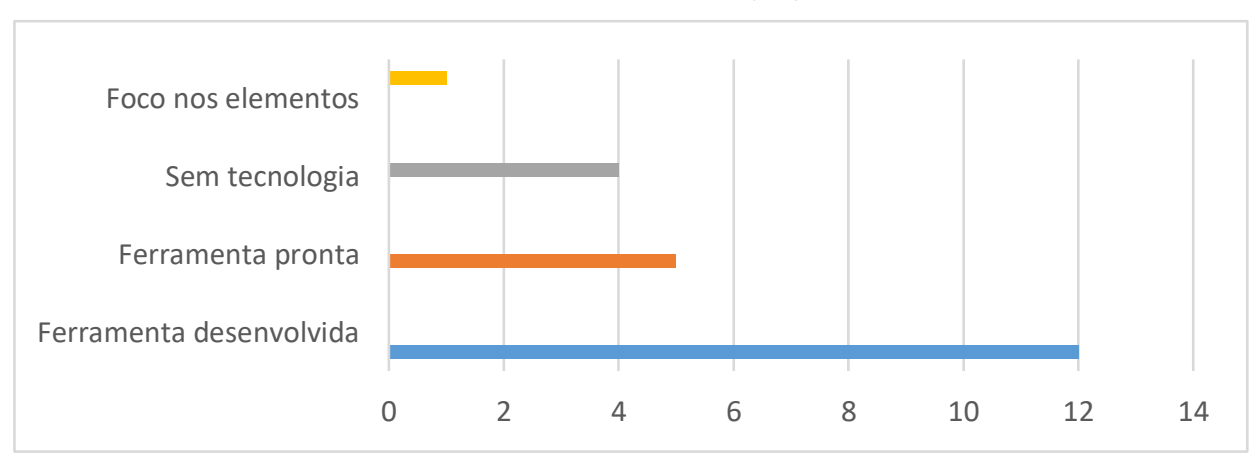

Figura 2. Estudos classificados pela base empírica

Também foi possível analisar as formas com que os autores escolheram para validar os argumentos, como mostra a Figura 3. Nota-se, que a maioria dos artigos demonstra que as pesquisas foram validadas em cursos superiores, aproximando conforme o resultado das pesquisas sobre gamificação aplicadas a educação de Borges et. al. (2013) e Regalado et. al. (2015). Acredita-se que essa validação sucede na maioria das vezes pelos estudantes aplicarem com mais facilidade a pesquisa na própria universidade. Após a essa ênfase, foi possível perceber que os artigos são diversificados de acordo com a validação e conclusão. Aproxima-se o trabalho de Ogawa et. al. (2015), o qual também apresentou suas análises de gamificação. Em três trabalhos os próprios autores realizaram avaliações das ferramentas e abordaram suas discussões. $\mathrm{Na}$ pesquisa de Silva et. al. (2016), os autores, também validaram com o grupo de pesquisa envolvido. As outras pesquisas apresentaram a validação realizada no ensino médio 
(Neto et. al., 2015), ensino fundamental (Seixas et. al., 2014; Pedro et. al., 2016), formulário compartilhado com o público (Lopes et. al., 2015) e, em um estudo foi desenvolvida uma plataforma para deficiente intelectual, validada com os mesmos sujeitos (Colpani et. al., 2016).

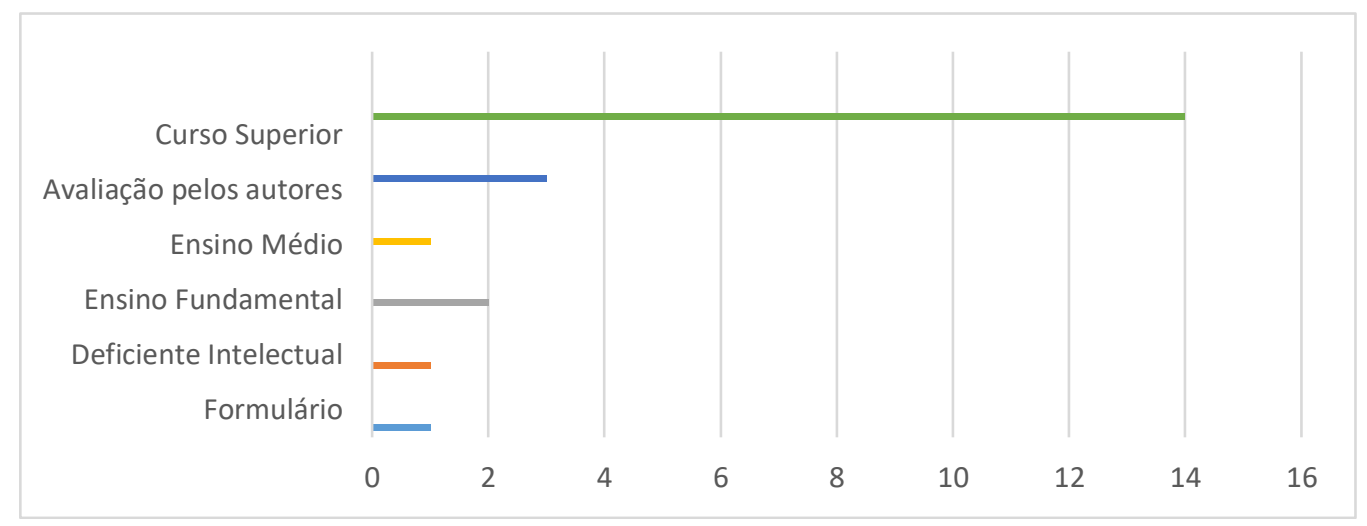

Figura 3. Estudos classificados pela validação da base empírica

A maioria dos trabalhos discutiu aplicação de base empírica, como já mencionado, com o objetivo de refletir sobre os benefícios das técnicas da gamificação, contribuição no processo de ensino, engajamento dos alunos, realização de atividades participativas e, ainda, mais interativas. O objetivo foi tornar os alunos mais interessados despertando a criatividade. Após as análises dos artigos, alguns objetivos semelhantes do usa da gamificação foram encontrados: (1) Aperfeiçoar habilidades; (2) Engajar e motivar alunos em atividades na sala de aula e no ambiente de aprendizagem; (3) Aprimorar a forma do aprendizado; (4) Propor desafios para contribuir no processo de ensino-aprendizagem; (5) Ocasionar mudança de comportamento; e (6) Promover mecanismos de socialização e aprendizado em grupo.

A nuvem de palavras, conforme Figura 4, gerada na ferramenta WordClouds ${ }^{1}$, apresenta as palavras mais frequentes de um texto, nesse caso, são os termos mais utilizadas nos artigos analisados. Palavras mais frequentes são apresentadas em fontes de tamanho maior, diminuindo a fonte conforme a frequência. Como é possível verificar, as pesquisas relatam em utilizar os elementos de jogos, como, feedback, desafios, para motivação e engajamentos dos alunos, aperfeiçoando suas habilidades.

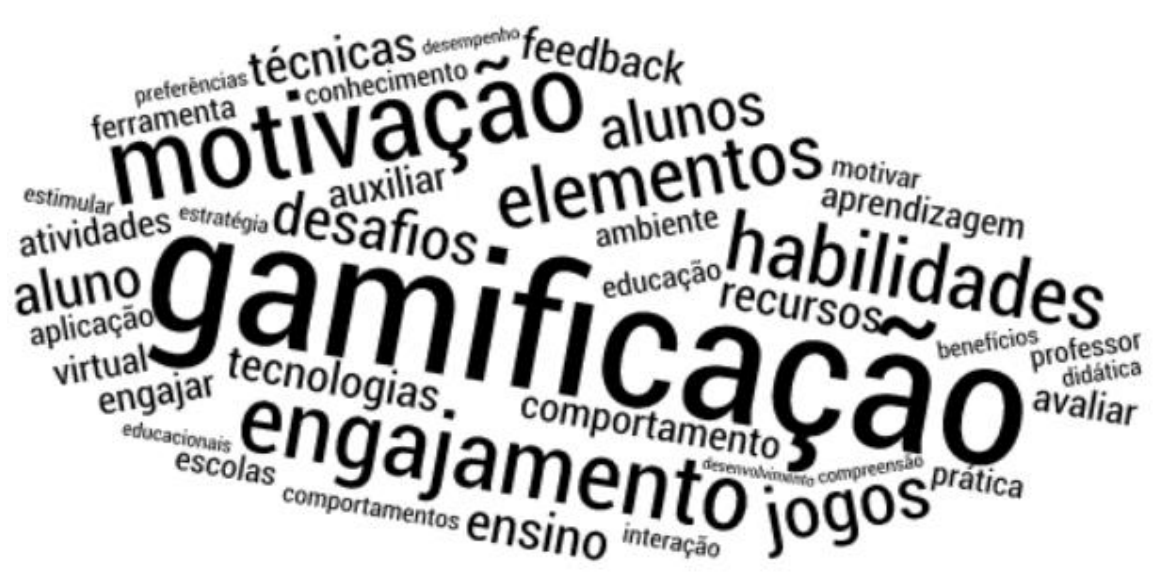

Figura 4. Nuvem de palavras

\footnotetext{
${ }^{1} \mathrm{http}: / /$ www.wordclouds.com/
} 
Relembrando as questões de pesquisas elaboradas: (1) As técnicas de gamificação auxiliam no processo de ensino e aprendizagem? (2) Quais são as técnicas de gamificação utilizadas no processo de ensino e aprendizagem?, pode-se afirmar que esse mapeamento sistemático da literatura obteve as respostas das mesmas. Por meio das análises dos artigos, é possível afirmar que a gamificação contribui para o processo de ensino e aprendizagem em vários contextos e conteúdo, mas não substitui a forma tradicional, auxiliando dentro e fora da sala de aula para a construção do conhecimento. Também, identificou-se nos artigos quais são os elementos de jogos mais utilizados no processo de gamificação aplicada à educação:

Tabela 5. Elementos de jogos encontrados nos artigos revisados

\begin{tabular}{|l|l|}
\hline \multicolumn{1}{|c|}{ Elemento } & \multicolumn{1}{c|}{ Descrição } \\
\hline Desafios/Missões & $\begin{array}{l}\text { Tarefa que exige esforço para resolver, com } \\
\text { objetivo e recompensa. }\end{array}$ \\
\hline Feedback & Informações sobre como o jogador está atuando. \\
\hline Recompensas/Medalhas & Benefício para alguma ação ou conquista. \\
\hline Pontos & $\begin{array}{l}\text { Ações no jogo que atribuem pontos. Semelhante a } \\
\text { recompensas/medalhas. }\end{array}$ \\
\hline Níveis & $\begin{array}{l}\text { Representação numérica da evolução do jogador/ } \\
\text { aluno. }\end{array}$ \\
\hline Rankings/Placar & $\begin{array}{l}\text { Classificação dos jogadores/alunos de acordo com } \\
\text { a pontuação. }\end{array}$ \\
\hline Narrativa & Um enredo consistente e contínuo. \\
\hline Restrições & $\begin{array}{l}\text { Refere-se à limitação da liberdade dos jogadores } \\
\text { dentro do jogo. }\end{array}$ \\
\hline
\end{tabular}

É importante mencionar que esses elementos de jogos apresentados são os mais utilizados nas pesquisas selecionadas na revisão da literatura, mas a gamificação utiliza uma série de outros elementos e estratégias de jogos. Muitos artigos observaram que a aplicação desorientada do uso desses elementos pode desmotivar os alunos e comprometer os resultados. Esse mapeamento alcançou os dois objetivos específicos propostos no início do processo: (1) Localizar trabalhos relacionados ao assunto gamificação na área da educação e (2) Desenvolver conhecimento sobre a fundamentação teórica da gamificação. Com este estudo localizou-se 22 artigos e com isso foi possível o desenvolvimento do conhecimento da gamificação e seus aspectos, quais elementos estão sendo mais utilizados para uma aplicação no processo de ensinoaprendizagem

\section{Considerações finais}

Este artigo apresentou como objetivo a realização de um mapeamento sistemático da literatura aplicada à educação, proporcionando apresentar e discutir os trabalhos que estão sendo desenvolvidos em pesquisas. Para alcançar esse objetivo foi proposto um processo de revisão, tal que, inicialmente, foram definidos objetivos gerais e questões de pesquisa. $\mathrm{O}$ estudo foi realizado baseado no objetivo de um projeto, em andamento, em que os autores investigam elementos de jogos utilizados no processo de gamificação em AVAs. De acordo com os resultados obtidos, é possível afirmar que o intento da revisão foi alcançado e que as questões de pesquisas foram respondidas, na medida em que se proporcionou uma análise e discussão dos artigos relacionados. A análise e 
discussão foram realizadas em acordo com o ano de publicação, base empírica e validação do estudo de caso.

De acordo com os artigos analisados compreende-se que a busca por inovações capazes de promover motivação e engajamento dos alunos é uma constante semelhança entre eles. Foi possível perceber que as pesquisas de gamificação na educação são recentes, pois se comprova que dos 22 artigos selecionados, 12 são do ano de 2016, mostrando, assim, que a perspectiva de crescimento aumente a cada ano. Há inúmeros elementos de jogos que podem ser utilizados no processo de gamificação, mas por meio dessa análise foi possível descobrir aqueles que estão sendo mais utilizados na educação: Desafios/Missões, Feedback, Recompensas/Medalhas, Pontos, Níveis, Rankings/Placar, Narrativa e Restrições. Outro fator em destaque, é que na maioria dos artigos os autores desenvolveram suas próprias ferramentas e implementaram elementos de jogos para validar a gamificação. Enfatiza-se, ainda, que na maioria dos trabalhos o estudo de caso foi realizado no ensino superior. Outro ponto a ser ressaltado é que todos os artigos, independentemente da base empírica, o modo de validação ou os elementos de jogos selecionados para o processo de gamificação, avaliaram os benefícios da gamificação, abordando, ainda, que esse processo melhora o desempenho dos alunos participantes no processo de ensino-aprendizagem. Esse indicador corrobora com a afirmativa de que a utilização da gamificação como estratégia didática contribui positivamente no processo de ensino.

\section{Referências bibliográficas}

ALMEIDA, C., VALE, L., CUNHA, R., \& GOMES, L. (2016). Avaliação do processo de Gamificação acerca do tema Direitos Humanos. In: Anais do Workshop de Informática na Escola (Vol. 22, No. 1, p. 379).

ANDRADE, J. O. \& CANESE, M. (2013). Um Sistema Web Gamificado para a Aprendizagem de Lógica Formal. In: Simpósio Brasileiro de Informática na Educação-SBIE (Vol. 24, No. 1, p. 426).

BORGES, S. D. S., REIS, H. M., DURELLI, V. H., BITTENCOURT, I. I., JAQUES, P. A., \& ISOTANI, S. (2013). Gamificação aplicada à educação: um mapeamento sistemático. In: Simpósio Brasileiro de Informática na Educação-SBIE (Vol. 24, No. 1, p. 234).

COLPANI, R. \& HOMEM, M. R. P. (2016). Realidade Aumentada e Gamificação na Educação: uma aplicação para auxiliar no processo de aprendizagem de alunos com deficiência intelectual. RBIE, 24 (1).

DA SILVA, A. R., ESTRUC, M., \& PIMENTEL, M. (2016). Uso da Inteligência Coletiva para Identificação de Mensagens Relevantes em um Bate-papo Gamificado. In: Simpósio Brasileiro de Informática na Educação-SBIE (Vol. 27, No. 1, p. 1265).

DE FREITAS, S., LIMA, T., CANEDO, E., \& COSTA, R. L. (2016). Gamificação e avaliação do engajamento dos estudantes em uma disciplina técnica de curso de graduação. In: Simpósio Brasileiro de Informática na Educação-SBIE (Vol. 27, No. 1, p. 370).

DOMÍNGUEZ, A., SAENZ-DE-NAVARRETE, J., DE-MARCOS, L., FERNÁNDEZSANZ, L., PAGÉS, C., \& MARTÍNEZ-HERRÁIZ, J. J. (2013). Gamifying learning experiences: Practical implications and outcomes. Computers \& Education, 63, 380392. 
FALCÃO, A. P., LEITE, M. D., \& TENÓRIO, M. M. (2014). Ferramenta de apoio ao ensino presencial utilizando gamificação e design de jogos. In: Simpósio Brasileiro de Informática na Educação-SBIE (Vol. 25, No. 1, p. 526).

FARDO, M. L. (2013). A gamificação aplicada em ambientes de aprendizagem. RENOTE, 11(1).

FARIA, V. P., COSTA, H., \& JÚNIOR, P. P. (2016). eQuest: Um Sistema de Resposta para Estudantes Gamificado. In: Anais dos Workshops do Congresso Brasileiro de Informática na Educação (Vol. 5, No. 1, p. 280).

FERREIRA, L., INOCÊNCIO, A. C., JÚNIOR, P. A. P., \& LOPES, M. M. (2016). Gamificação Aplicada ao Ensino de Gerência de Projetos de Software. In: Anais do Workshop de Informática na Escola (Vol. 22, No. 1, p. 151).

KAPP, K. M. (2012). The gamification of learning and instruction: game-based methods and strategies for training and education. John Wiley \& Sons.

KITCHENHAM, B. A. (2004). Procedures for Performing Systematic Reviews. Keele, UK, Keele University,

KLOCK, A. C. T., DE CARVALHO, M. F., ROSA, B. E., \& Gasparini, I. (2014). Análise das técnicas de Gamificação em Ambientes Virtuais de Aprendizagem.

RENOTE, 12(2).

KLOCK, A. C. T., DA CUNHA, L. F., \& GASPARINI, I. (2015). Um modelo conceitual para a gamificação de Ambientes Virtuais de Aprendizagem. RENOTE, 13(1).

LOPES, R. A., TODA, A. M., \& BRANCHER, J. D. (2015). Um estudo preliminar sobre elementos extrínsecos e intrínsecos do processo de Gamification. RBIE, 23(3).

MAEKAWA, C., NAGAI, W., \& IZEKI, C. (2015). Relato de Gamificação da disciplina Projeto e Análise de Algoritmos do curso de Engenharia de Computação. In: Anais dos Workshops do Congresso Brasileiro de Informática na Educação (Vol. 4, No. 1, p. 1425).

MANCINI, M. C., \& SAMPAIO, R. F. (2007). Estudos de revisão sistemática: um guia para síntese criteriosa da evidência científica. Rev bras fisioter, 11(1), 83-9.

NAGAI, W., \& IZEKI, C. (2016a). As estratégias de gamificação da disciplina de Projeto e Análise de Algoritmos segundo o Modelo Dinâmico de Aprendizado baseado em Jogos. In: Anais dos Workshops do Congresso Brasileiro de Informática na Educação (Vol. 5, No. 1, p. 1159).

NAGAI, W., IZEKI, C., \& DIAS, R. (2016b). Experiência no Uso de Ferramentas Online Gamificadas na Introdução à Programação de Computadores. In: Anais do Workshop de Informática na Escola (Vol. 22, No. 1, p. 301).

NETO, A., DA SILVA, A. P., \& BITTENCOURT, I. I. (2015). Uma análise do impacto da utilização de técnicas de gamificação como estratégia didática no aprendizado dos alunos. In: Simpósio Brasileiro de Informática na Educação- SBIE (Vol. 26, No. 1, p. 667).

OGAWA, A. N., MAGALHÃES, G. G., KLOCK, A. C. T., \& GASPARINI, I. (2015). Análise sobre a gamificação em Ambientes Educacionais. RENOTE, 13(2).

PEDRO, L., \& ISOTANI, S. (2016). Explorando o Impacto da Gamificação na Redução do Gaming the System em um Ambiente Virtual de Aprendizagem. In: Anais dos 
Workshops do Congresso Brasileiro de Informática na Educação (Vol. 5, No. 1, p. 81).

PETERSEN, K., FELDT, R., MUJTABA, S. \& MATTSSON, M. (2008) Systematic Mapping Studies in Software Engineering. In: Proceedings of the international conference on Evaluation and Assessment in Software Engineering (Vol. 8, p. 6877).

RAMOS, S., \& PIMENTEL, E. P. (2015). VirtuaLabQ Ambiente Gamificado para a Prática Experimental de Transformações Químicas. In: Simpósio Brasileiro de Informática na Educação-SBIE (Vol. 26, No. 1, p. 587).

RAPOSO, E. H. S., \& DANTAS, V. (2016). O Desafio da Serpente-Usando gamification para motivar alunos em uma disciplina introdutória de programação. In: Simpósio Brasileiro de Informática na Educação-SBIE (Vol. 27, No. 1, p. 577).

REGALADO, M. R., DA SILVA, T. R., \& DA SILVA ARANHA, E. H. (2015). Um Mapeamento Sistemático sobre o uso da Gamificação em Ambientes Virtuais de Aprendizagem. RENOTE, 13(2).

SEIXAS, L. da R., GOMES, A. S., MELO FILHO, I. J. \& RODRIGUES, R. L. (2014). Gamificação como Estratégia no Engajamento de Estudantes do Ensino Fundamental. In: Simpósio Brasileiro de Informática na Educação-SBIE (Vol. 25, No. 1, p. 559).

SILVA, T., MELO, J., \& TEDESCO, P. (2016). Um modelo para promover o engajamento estudantil no aprendizado de programação utilizando gamification. In: Anais dos Workshops do Congresso Brasileiro de Informática na Educação (Vol. 5, No. 1, p. 71).

TODA, A., RAFAEL, Y., CRUZ, W., XAVIER, L., \& ISOTANI, S. (2016). Um processo de Gamificação para o ensino superior: Experiências em um módulo de Bioquímica. In: Anais do Workshop de Informática na Escola (Vol. 22, No. 1, p. 495).

ZICHERMANN, G., \& CUNNINGHAM, C. (2011). Gamification by design: Implementing game mechanics in web and mobile apps. O'Reilly Media, Inc. 